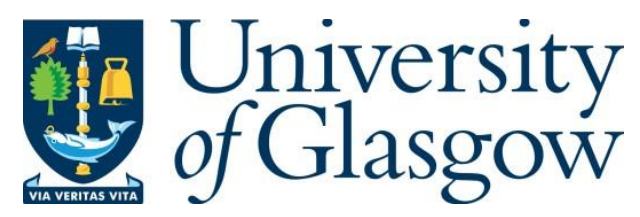

Zhao, P., Li, S., Feng, X., Barnett, S. M., Zhang, W., Cui, K., Liu, F. and Huang, Y. (2019) Universal linear optical operations on discrete phase-coherent spatial modes with a fixed and non-cascaded setup. Journal of Optics, 21(10), 104003. (doi:

$10.1088 / 2040-8986 / a b 3 d 8 b)$

There may be differences between this version and the published version. You are advised to consult the publisher's version if you wish to cite from it.

http://eprints.gla.ac.uk/197305/

Deposited on: 23 September 2019

Enlighten - Research publications by members of the University of Glasgow http://eprints.gla.ac.uk 


\title{
Universal linear optical operations on discrete phase-coherent spatial modes with fixed and non-cascaded setup
}

\author{
Peng Zhao ${ }^{1}$, Shikang $\mathbf{L i}^{1}$, Xue Feng ${ }^{1, *}$, Stephen M. \\ Barnett $^{2}$, Wei Zhang ${ }^{1}$, Kaiyu Cui ${ }^{1}$, Fang Liu ${ }^{1}$, Yidong \\ Huang $^{1}$ \\ ${ }^{1}$ Department of Electronic Engineering, Tsinghua University, Beijing 100084, \\ China. \\ ${ }^{2}$ School of Physics and Astronomy, University of Glasgow, Glasgow G12 8QQ, \\ United Kingdom. \\ E-mail: $x$-feng@tsinghua.edu.cn
}

March 2019

\begin{abstract}
Linear optical operations are fundamental and significant for both quantum mechanics and classical technologies. We demonstrate a non-cascaded approach to perform arbitrary linear operations for $\mathrm{N}$-dimensional phase-coherent spatial modes using meticulously designed phase gratings. As implemented on spatial light modulators (SLMs), the unitary transformation matrix has been realized with dimensionalities ranging from 7 to 24 and the corresponding fidelities are from $95.1 \%$ to $82.1 \%$. For the non-unitary operators, a matrix is presented for the tomography of a 4-level quantum system with a fidelity of $94.9 \%$. Thus, the linear operator has been successfully implemented with much higher dimensionality than that in previous reports. It should be mentioned that our approach is not limited to SLMs and can be easily applied on other devices. Thus our proposal could provide another option to perform linear operations with a simple, fixed, error-tolerant and scalable scheme.
\end{abstract}

Submitted to: J. Phys. B: At. Mol. Opt. Phys. 


\section{Introduction}

Linear operations on an $N$-dimensional vector are a powerful tool both for quantum optics and for classical optical information processing. In the quantum domain, several information protocols have been demonstrated with linear optics. These include the famous KLM scheme for universal quantum computing [1], boson sampling $[2,3,4,5,6,7,8,9]$, quantum gates and Hadamard operations [8], quantum walks [10], Quantum transport [11], homomorphic encryption [12] and quantum metrology [13]. For classical information, linear optics has been applied for the programmable filters for microwave signals [14], photonic switch matrices for packet data networks [15] and optical neural network for vowel recognition [16].

Typically, arbitrary linear operators can be achieved with a programmable optical multiport interferometer introduced by Reck et.al. [17]. In the Reck scheme, the $N \times N$ transformation matrix is achieved by a specific triangular mesh of $2 \times 2$ beam splitters (or directional coupler) and phase shifters. Recently, some modified designs of the Reck scheme have been proposed to achieve more compact and loss-tolerant multiport interferometer [18], non-unitary linear operations [19] and an on-chip multiple interferometer has been developed to unscramble beams [20]. However, both the Reck scheme and variants of it require, in general, $N(N-1) / 2$ beam splitters (or directional couplers) and a corresponding number of phase shifters. Thus, as the dimensionality $(N)$ increased, the complexity in terms of system arrangement and parameter control would grow significantly as $\mathrm{O}\left(N^{2}\right)$. Consequently, realising a high-dimensional transformation matrix is still very technologically challenging. To our knowledge only a $6 \times 6$ unitary transformation matrix has been implemented in the Reck scheme [8] while the achievable values are $9 \times 9$ [9], $13 \times 13$ [6], $15 \times 15$ [21] and $26 \times 26$ [11] for constant and partially adjustable matrix elements, respectively.

Recently, some linear transformation schemes have been proposed within the orbital angular momentum (OAM) and frequency domains. In the OAM domain, high-dimensional transformations have not been achieved yet [22]. For transformation in the frequency domain, multi-beam splitting and recombining are employed. The dimensionality of such approach relies on high speed phase modulator, which is still technically challenging. Meanwhile, the cascaded structure is still required to achieve both high dimensionality and nearunitary efficiency [23]. Apart from the approaches implemented with OAM modes or in frequency domain, generating a chosen unitary transformation has also been demonstrated using multiple spatial light modulators [24] and this technique has been used as a multiplexer [25].
Here we propose and demonstrate a simple, fixed, error-tolerant and scalable scheme based on meticulously designed phase gratings in order to perform arbitrary linear operations for $N$-dimensional phasecoherent spatial modes. In contrast with the Reck scheme, a cascaded multi-stage mesh is avoided and any linear operator can be decomposed into just two processes, namely beam splitting and recombining. This simplification is comparable to the ability to sort OAM modes with just two specially designed elements [26] in place of the multiple interferometers in an earlier device [27]. Our proposal applies, at least in principle, irrespective of the number of modes, $N$, that are introduced. In our experiment, implemented on a spatial light modulator (SLM), the unitary transformation matrix has been realized with dimensionalities ranging from 7 to 24 with corresponding fidelities from $95.1 \%$ to $82.1 \%$. Besides the unitary operations, non-unitary operators can also be implemented, which makes it more flexible for certain applications. As a concrete example, a $4 \times 16$ matrix is presented for the tomography of a 4-level quantum system, performed with a fidelity of $94.9 \%$. An additional feature of our proposal is that the high-dimensional states can be coded with any designed optical modes, including the fundamental Gaussian modes, which is usually quite desired in quantum applications to improve the collection efficiency. These results indicate that the linear operator has been successfully implemented with much higher dimensionality than in previous reports. Although our proposal is not intrinsically lossless for an arbitrary linear operator, we have provided an optimization process to map the matrix elements into the phase grating patterns with the lower bound of optimized transformation efficiency of $1 / \sqrt{N}$ while the achieved value is $\sim 0.8 / \sqrt{N}$ in our experiments.

Finally, our proposal is an approach employing the spatial degree of freedom so that there are some known advantages. In particular, the identity of the photons could be readily maintained and the manipulation is more manageable. Thus, spatial modes are adopted in many quantum applications including information processing $[28,29,30,31]$ and measuring Winger function of a quantum-chaotic system [32]. Furthermore, there is the potential to combine our approach with that in the frequency domain since the spatial distribution and optical frequency are independent degrees of freedom. Thus our proposal could provide a feasible approach to perform linear operations with optical modes.

\section{Results}

\section{1. $N$-dimensional optical states}

As a general consideration, our task is to perform a linear transformation on the $N$-dimensional vector of 


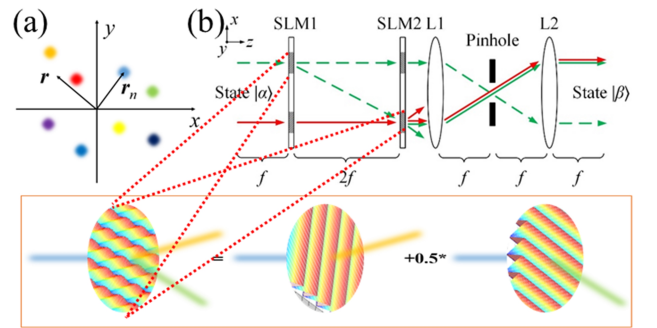

(c)

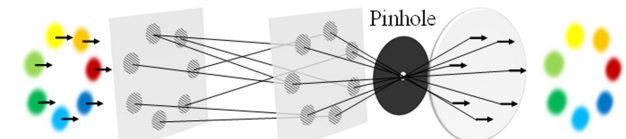

$|\alpha\rangle$ State

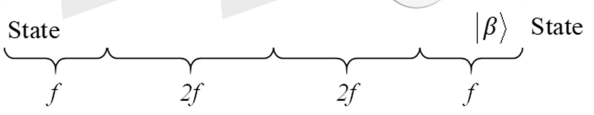

(d)

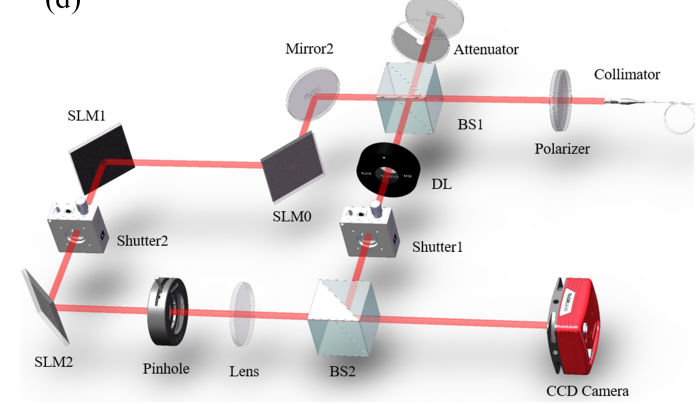

Figure 1. The operation principle and the experimental setup of the linear transformation on the high-dimensional optical states. (a) The high-dimensional optical states is encoded with phasecoherent spatial modes. (b) Beam splitting and recombining are achieved with the phase gratings, which can be achieved by using SLMs. (c) 3-D view of full scheme of the state transformation, including two SLMs, a pinhole and a lens. (d) Experimental setup of the linear transformation on discrete phase-coherent spatial modes. BS: beam splitter, SLM: spatial light modulator, DL: delay line.

$|\alpha\rangle$ to obtain an $M$-dimensional vector of $|\beta\rangle$. In general, this entails realizing a complex matrix $\boldsymbol{T}(M \times N)$ such that $|\beta\rangle=\boldsymbol{T}|\alpha\rangle$. Here the matrix $\boldsymbol{T}$ could be either unitary or non-unitary. Inspired by structural light beams, the state vector can be represented with optical phase-coherent spatial modes. To form an $N$ dimensional vector of $\left[\alpha_{1}, \alpha_{2}, \cdots, \alpha_{N-1}, \alpha_{N}\right]^{T}$, discrete beams are employed, as shown in Fig. 1(a). The complex field amplitude of each optical beam represents a corresponding coefficient of $\alpha_{n}$. These beams have to be phase-coherent so that complex operation can be performed. Furthermore, the employed modes should share the same profile shape so as to allow them to interfere. Here, discrete Gaussian beams are employed to represent the state vectors:

$$
\begin{aligned}
& |\alpha\rangle=\sum_{n}^{N} \alpha_{n} u\left(\boldsymbol{r}-\boldsymbol{r}_{n}\right) \\
& \left.u\left(\boldsymbol{r}-\boldsymbol{r}_{n}\right)\right|_{z=0}=u_{0} \exp \left(-\frac{\left|\boldsymbol{r}-\boldsymbol{r}_{n}\right|^{2}}{w_{0}^{2}}\right),
\end{aligned}
$$

where $\boldsymbol{r}$ and $\boldsymbol{r}_{n}$ denote the position vector and the central position of the $n$th Gaussian beam spot, respectively, while $u_{0}$ is the normalization coefficient. To ensure the mode overlap small enough, the distance between the different spots should be much larger than the waist size of $w_{0}\left(w_{0} \ll\left|\boldsymbol{r}_{i}-\boldsymbol{r}_{j}\right|\right)$ and the detailed discussion is given in the supplementary material (S6).

\subsection{Linear operations with discrete phase-coherent spatial modes}

In the Reck scheme, any linear unitary operator can be decomposed into a series of 2-dimensional beam splitting and recombining operations, with the transformation coefficients controlled by inserting phase shifters. As a consequence, $N(N-1) / 2$ units are required for full generality. In our scheme, the splitting and recombining of the Gaussian beams are based on a series of phase gratings implemented on two SLMs as proposed in our previous work [33]. An SLM is an efficient and programmable device, which is capable of generating arbitrary beams and so is ideal for our task [34]. As shown in Fig. 1(b), the input vector $|\alpha\rangle=\sum_{n}^{N} \alpha_{n} u\left(\boldsymbol{r}-\boldsymbol{r}_{n}\right)$ is incident on the SLM1, on which a diffraction pattern is pre-settled to mimic a series of blazed gratings so that each Gaussian beam can be split into $M$ beams with a selected ratio and then incident on SLM2. On SLM2, there is also a properly prepared diffraction pattern so that the split modes are recombined with selected weights. It should be noted that each of the reformed beam spots on SLM2 is a superposition of several tilted beams diffracted from different spots on SLM1. Thus, there are some undesired side lobes in addition to the desired recombined one. To eliminate these side lobes and to keep the output beam propagation direction as well as the original state, a $2 f$ system with a pinhole is employed for realignment and spatial filtering. Following this, the output vector of $|\beta\rangle=\sum_{m}^{M} \beta_{m} u\left(\boldsymbol{r}-\boldsymbol{R}_{m}\right)$ is obtained, where $\boldsymbol{R}_{m}$ denotes center position of the $m$ th Gaussian beam. In our approach, the most important issue is how to map the complex matrix elements onto the phase gratings on the SLM1 and SLM2. The modulation function on $n$th spot of SLM1 and $m$ th spot of SLM2 are settled as: 


$$
\begin{aligned}
& H_{\text {diff } 1}=\arg \left\{\sum_{m}^{M} a_{m n} \exp \left[i \boldsymbol{k}_{m n} \cdot\left(\boldsymbol{r}-\boldsymbol{r}_{n}\right)\right]\right\} \\
& H_{\text {diff }}=\arg \left\{\sum_{n}^{N} b_{m n} \exp \left[-i \boldsymbol{k}_{m n} \cdot\left(\boldsymbol{r}+\boldsymbol{R}_{m}\right)\right]\right\}
\end{aligned}
$$

where the coefficients of $a_{m n}$ and $b_{m n}$ are the beam splitting and recombining weight from $n$th spot on SLM1 to $m$ th spot on SLM2, respectively. The diffraction coefficients of $\boldsymbol{k}_{m n}$ are determined by the tilt angle. Additionally, some auxiliary holograms are added in order to compensate for the divergence during the propagation of Gaussian beams. A detailed description can be found in section of S.1 S.3 in the supplementary material. Here only the key points are presented. For a target transformation matrix of $\boldsymbol{T}$ (elements of $t_{m n}$ where $n=1, . ., N$ and $m=$ $1, . ., M)$, the coefficients of $a_{m n}$ and $b_{m n}$ should be determined according to the relation of $a_{m n} \cdot b_{m n}=$ $t_{m n}$. In principle, an arbitrary coefficient pair of $\left(a_{m n}, b_{m n}\right)$ can satisfy such relation to perform the linear operation. However, different strategies for determining the coefficient pair result in different efficiencies for implementing the matrix $\boldsymbol{T}$. Due to the passive property of SLMs and spatial filtering of pinhole, there is some energy loss when processing the optical states. Thus, the actually obtained matrix of $\boldsymbol{T}^{\prime}$ may have an overall energy loss compared to the ideal target matrix of $\boldsymbol{T}$ (denoted as $\boldsymbol{T}^{\prime}=\eta \boldsymbol{T}$ ). To account for this, we introduce the parameter of $\eta=\boldsymbol{T}^{\prime} / \boldsymbol{T}$, which characterizes the efficiency of implementation. To maximize this efficiency, the coefficient pair of $\left(a_{m n}, b_{m n}\right)$ is obtained using Lagrange's method. A detailed discussion of this is provided in S.1 in the supplementary material. Our theoretical analysis indicates that the efficiency of implementation is about $\eta \approx 1 / \sqrt{N}$ for an $N$-dimensional unitary matrix transformation. In our experiments, the achieved efficiency is about $\eta \approx 0.8 / \sqrt{N}$. A detailed discussion may be found in the results section.

\subsection{Optical setup}

Figure 1(d) shows the experimental setup, in which there are three parts for generating $N$-dimensional input vector, performing the linear operations, and measuring the $M$-dimensional output vector. The incident light beam is emitted from a continuous wave laser with operation wavelength of $1550 \mathrm{~nm}$ and linewidth of $1 \mathrm{kHz}$ (Rio Orion). It should be mentioned that a narrow linewidth light source is required since coherent states are employed in our proposed scheme. The SLM0 is employed to generate the input vector by modulating the incident Gaussian beam with the same beam splitting holograms, while the SLM1, SLM2, pinhole and (a)
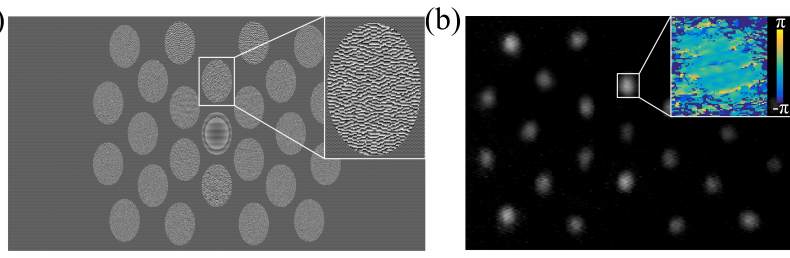

(c)
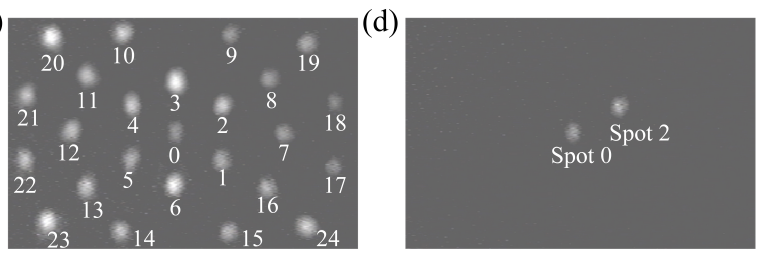

(e)
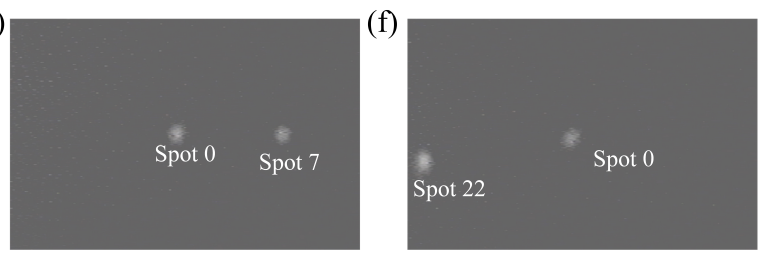

Figure 2. Typical holograms and measured high-dimensional optical states. (a) The hologram on SLM1 for a 24-dimensional unitary linear transformation. (b) The intensity of 24dimensional optical states of vector $[1,1,1, \cdots, 1]^{T}$ is recorded with CCD camera while the right corner shows the measured phase of one light spot in the state. (c) The relation between the state vector and the light spots. There are 24 light spots for each vector labeled as spots $1 \sim 24$. Additionally, there is an extra light spot marked as spot 0 , which is introduced for the phase measurement. (d)-(f) Typical output after 24-dimensional unitary transformation. The input states are the column vectors of the conjugate transpose matrix of the matrix corresponding the unitary transformation. Thus the output states are the column vectors of the identity matrix, corresponding to the optical states with one spot is illumining. In (c)-(f), for clearer view, the brightness is increased by 20 percent compared to the raw data obtained by CCD camera.

the lens are utilized for the linear operation. As the linear operation is based on the phase-coherent modes, the phase terms of the output vector have to be measured. Thus, two beam splitters (BS1 and BS2) are inserted before SLM0 and CCD camera so that a typical Mach-Zender interferometer (MZI) is implemented to measure the phase terms with the method in our previous work [35]. The details can be found in S.4 of the supplementary material and the Ref. [35].

In our experiment, the employed SLMs (PLUTOTELCO013) are reflection type and the light beam is incident at $\sim 45$ degrees. Although the SLMs are considered as the transmission type for conveniently introducing the principle of operation is required, this does not affect the results significantly although some modification of the real implementation is necessary. Figure 2(a) shows a typical hologram on SLM1 for 24-dimensional coherent states. As the light beam is incident with $\sim 45^{\circ}$ on the SLMs, the hologram spot has to be distorted on purpose as an ellipsoid shape (shown in Fig. 2(a)) to 
keep the output spot circular in shape. For each hologram spot, the minor and major axes extend over about 70 pixels and 99 pixels, respectively, while the width of each pixel is 8 micrometers. As an SLM can only partially modulate the incident light beam, an additional phase grating with period of 4 pixels is introduced on each spot to separate directly reflected light beam from the modulated beam. Furthermore, the pixels out of the desired hologram spots should behave as the 'zero' modulation. Normally, the phase should be set as $\pi$ and zero alternatively. However, in our experiments, as the phase grating is introduced in each spot, a flat phase could also serve as the 'zero' modulation. Figure 2(b) shows the intensity of the output light spots recorded by a CCD camera. As mentioned above, the phase term of each spot is measured with the help of the MZI as well as CCD camera while the measured phase term of one spot is shown as the inset in Fig. 2(b). As shown in Fig. 2(b), there are some fluctuations on the phase profile, which come from the random variations of each arm in the MZI. Thus the phase term of each spot is calculated by averaging the overall phase profile to reduce the random fluctuations. Moreover, to deal with the unavoidable misalignment between different optical components, we have first measured the alignment error and modified the target matrix elements (especially the phase term). The detailed measurement and data processing approaches are provided in the supplementary material.

As shown in Fig. 2(c), (d) and (f), up to a 24dimensional linear transformations has been achieved, with the dimensionality limited by the resolution of SLMs. Some typical experimental results of 24dimensional transformation are shown in Fig. 2(c), (d) and (f) and a detailed discussion of these is provided in supplementary material. For the 24-dimensional case, the input states are settled as the column vectors of the conjugate transpose matrix of the matrix corresponding the unitary transformation, so that only the nth spot is illumining in the output vector. In Fig. 2(d) and (f), only the results for spot 2, 7 and 22 are shown while the full results are provided in supplementary material.

\subsection{Transformation fidelity}

For the linear operations, the most important parameter to evaluate the performance is the transformation fidelity:

$$
\text { fide }=\frac{\left|\sum_{m, n} t_{m n}^{\prime} \cdot t_{m n}^{*}\right|}{\sqrt{\sum_{m, n}\left|t_{m n}^{\prime}\right|^{2} \cdot \sum_{m, n}\left|t_{m n}\right|^{2}}},
$$

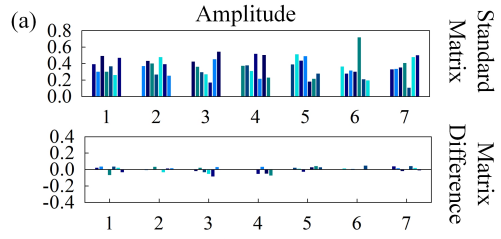

(b)
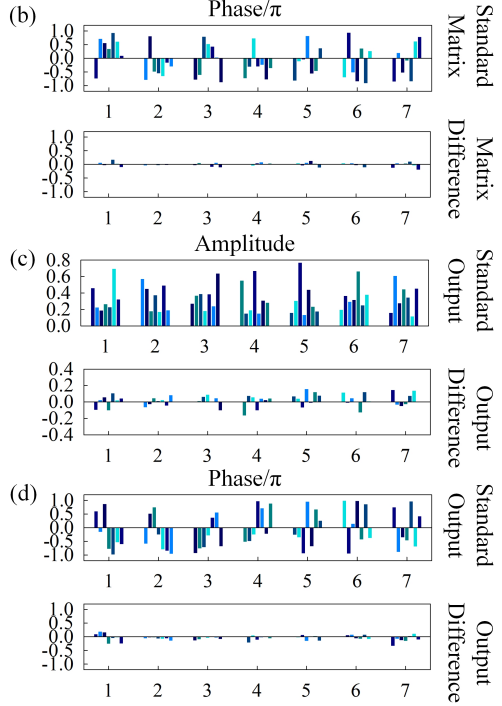

Figure 3. The elements of output vector after a randomly generated 7-dimensional unitary matrix acting on each column vector of the unit matrix ( $\mathrm{a}$ and $\mathrm{b}$ ) and the DFT matrix (c and d). The upper and bottom figures show the target elements and differences between the measured elements, respectively. (a), (c), The amplitude term. (b), (d), The phase term.

where $t_{m n}^{\prime}$ and $t_{m n}$ denote the matrix elements of obtained and target transformation, respectively. It is this fidelity that quantifies the precision of the transformation. To obtain the matrix elements, each column vector of the identity matrix is selected in turn as the input state in turn so that each column of the matrix can be obtained. The details are provided in the supplementary material. For simplicity, instead of 24-dimensional transformations, a 7-dimensional matrix is implemented and measured to investigate the transformation fidelity.

Firstly, the random unitary matrices are obtained as the results of singular value decomposition of randomly generated matrices, in which each complex element inside have random amplitude $(0 \sim 1)$ and phase terms $(0 \sim 2 \pi)$ with uniform probability distributions. A 7-dimensional unitary matrix and the corresponding measurement results are shown in Fig. 3(a) and (b), in which the horizontal coordinate is the index of the column vector in the matrix. With Equ. (3), the fidelity is calculated as high as $97.7 \%$.

Although, employing the column vector of unit matrix as input is a quite direct way to measure the matrix elements, the obtained value is sometimes not perfectly accurate as the beam recombination may not be in- 
volved fully. Thus, the input vector should have multiple nonzero values. Especially, if each element in the input vector has the same absolute value, in which case the full beam recombination is involved. To this end, we enact the transformation on each column vector of the discrete Fourier transformation (DFT) matrix as the input vectors. The target/difference output vectors for these are shown in Fig. 3(c) and (d).

Mathematically, the inner product between the outputs scanned by column vectors in an arbitrary unitary matrix would be the same as the one scanned by column vectors in unit matrix, and the deduction can be found in supplementary material. Thus, the inner product between the outputs scanned with vectors in DFT matrix could also present the transformation fidelity and the value is calculated as high as $95.1 \%$, which is only a little degraded to that obtained from the directly measuring the matrix elements $(97.7 \%)$. This result indicates that the beam combining is quite accurate and our proposed approach achieves a high fidelity. Randomly generated unitary matrices with higher dimensionalities of 16,19 and 24 have been implemented and the measured values of the corresponding transformation fidelities are $85.2 \%, 83.9 \%$, and $82.1 \%$, respectively. It should be mentioned that, for such high-dimensional transformations, a projection method is adopted to measure the fidelities, while the detailed method and data are provided in supplementary material (S.5 and S.6).

It is our ambition to achieve the high-fidelity linear operation without the need for a multi-stage mesh. Thus, besides experimental demonstration, some simulations have been carried out to compare our proposal and the Reck scheme. The results are summarized in Fig. 4(a) (c). For most cases, the fidelity could be $\sim 99 \%$. The worst case is 2-dimension and the reason is that phase gratings behaves badly in small amount of fan-out, especially in this case. Thus, the fidelity actually increases along with the dimensionality at first and then drops slowly due to the increased complexity. An obvious advantage of our proposal is the noncascaded structure so that there are no accumulated implementing errors. In the Reck scheme, the fidelity of transformation would drop due to the inevitable imperfections associated with including large numbers of optical components, for example the losses in the beam splitters and the phase drift of the phase modulators. To deal with beam splitters losses, a modified Reck scheme [18] was proposed to achieve loss-tolerance by balancing the light path between different ports and our scheme has the same advantage. However, the modified Reck scheme still suffers from the phase drift, which could be introduced by the calibration error or the ambient fluctuations of the phase modulators, while our scheme has the advantage of drift-tolerance.
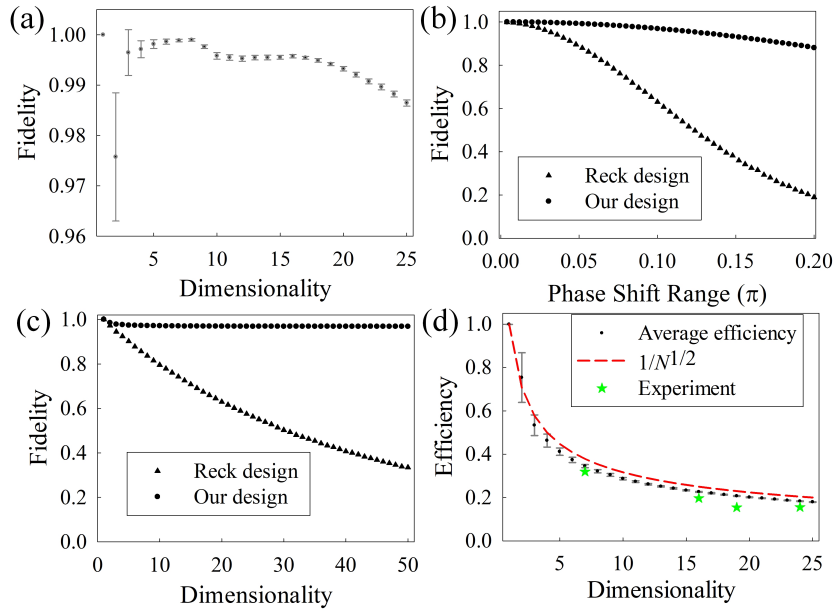

Figure 4. The comparison between simulations and experiments in terms of fidelities and efficiencies of the linear transformations. (a) The simulated transformation fidelity for 50 random unitary operations with dimensionality of $1 \sim 25$. (b) The simulated fidelity versus random phase shift ranges with 20dimensional transformation. (c) The simulated fidelity versus the dimensionality of transformation with random phase shift range of $\pi / 10$. (d) The simulated average efficiency for all unitary matrices versus matrix dimensionality is denoted as the black dot while the value of $1 / \sqrt{N}$ is also plotted as red line for comparison while the experimental values for 7-, 16-, 19- and 24-dimensional transformations are also denoted as green star.

Obviously, the impact of phase drift on Reck and our scheme is different. For comparisons, we assume that there is the same phase drift in Reck and our scheme. In the Reck scheme, the phase drift is added on each phase modulator, while in our scheme, it is added in each diffraction beam. Within the drift range, the phase value is considered as uniform probability distribution. Figure 4(b) and (c) are the calculated results of the fidelity versus phase drifts.

A further advantage of our proposal is that a nonunitary matrix and even a non-square matrix can readily be implemented. For instance, quantum tomography can be achieved by linear transformation with a $N \times N^{2}$ matrix, which can be readily implemented with our scheme. According to Ref. [36], the tomography of a 4-level quantum system can be achieved with a $4 \times 16$ matrix in Equ. (4).

Such a matrix has been implemented with fidelity of $95.3 \%$ measured by the direct elements scanning approach. This matrix could be treated as a combining of four square matrices with dimensionality of 4 . Thus, the fidelity could be obtained by inputting the column vectors of 4-dimensional DFT matrix four times and the value is as high as $94.9 \%$. This indicates that our proposal also works well for non-unitary operators. 
$\boldsymbol{T}=\left[\begin{array}{cccccccccccccccc}0 & 0 & 0 & 0 & 1 & 1 & 1 & 1 & 1 & 1 & 1 & 1 & 1 & 1 & 1 & e^{i \frac{2 \pi}{3}} \\ 1 & 1 & 1 & e^{i \frac{2 \pi}{3}} & 0 & 0 & 0 & 0 & 1 & e^{i \frac{2 \pi}{3}} & e^{-i \frac{2 \pi}{3}} & 1 & 1 & e^{i \frac{2 \pi}{3}} & e^{-i \frac{2 \pi}{3}} & 1 \\ 1 & e^{i \frac{2 \pi}{3}} & e^{-i \frac{2 \pi}{3}} & 1 & 1 & e^{i \frac{2 \pi}{3}} & e^{-i \frac{2 \pi}{3}} & e^{i \frac{2 \pi}{3}} & 0 & 0 & 0 & 0 & 1 & e^{-i \frac{2 \pi}{3}} & e^{i \frac{2 \pi}{3}} & 1 \\ 1 & e^{-i \frac{2 \pi}{3}} & e^{i \frac{2 \pi}{3}} & 1 & 1 & e^{-i \frac{2 \pi}{3}} & e^{i \frac{i \pi}{3}} & 1 & 1 & e^{-i \frac{2 \pi}{3}} & e^{i \frac{2 \pi}{3}} & e^{i \frac{2 \pi}{3}} & 0 & 0 & 0 & 0\end{array}\right]^{T}$

\subsection{Transformation efficiency}

In addition to the transformation fidelity, the transformation efficiency is an important parameter with which to evaluate the performance of linear operations. In the above section, about the transformation fidelity, the matrix elements as well as the output vectors are normalized so that the transformation efficiency has not been discussed. As mentioned, our scheme for arbitrary linear operator is not lossless due to the light beam filtering. The efficiency depends on the strategy to determine the splitting and recombining coefficients, which is discussed in supplementary material. The main point is the worst case will be touched for a non-sparse matrix, especially for the DFT matrix with value of $\eta \approx 1 / \sqrt{N}$ for $N$-dimensional transformation. For other types of linear operators, the transformation efficiency would be higher than this value. In particular, the Reck scheme can have a much higher efficiency. To verify the theoretical prediction, we have carried out some simulations with 50 random unitary-matrices for dimensionality of $1 \sim 25$ and Fig. 4(d) summarizes the results. The transformation efficiency is obtained by calculating the energy ratio between the output of target matrix and identity matrix while the error bars denotes the standard deviation of simulated efficiencies. It can be seen that the simulated efficiency is a little bit lower than the ideal prediction of $1 / \sqrt{N}$. The reason for this is that there is diffraction loss due beam splitting with the phase grating, which is not included in our theoretical analysis. To estimate the impact of diffraction loss, the ratio between the transmission efficiency obtained and the implementing efficiency from the resolve approach is calculated and the value is about 0.8 for transformation dimensionality of $7 \sim 25$. Thus, the transformation efficiency of our proposal could be estimated as $\eta \approx 0.8 / \sqrt{N}$ according to the simulations and experimental results.

\section{Discussion}

We have demonstrated a non-cascaded approach to perform arbitrary linear operations for $\mathrm{N}$-dimensional phase-coherent spatial modes. With meticulously designed phase gratings, not only unitary but also non-unitary operators can be implemented. The main features of our scheme are high-fidelity and error tolerance. According to the experiments implemented on SLMs, the transmission fidelity can be as high as $95.1 \%$ for randomly generated 7 -dimensional unitary matrix while the values are $82.1 \%$ and $94.9 \%$ for 24-dimensional unitary matrix and a $4 \times 16$ matrix (for the tomography of a 4-level quantum system), respectively. Moreover, although the phase gratings are implemented on a SLM in this work, our approach is not limited to SLMs and can be easily applied on other devices. Thus we believe that our proposal provides another option to perform linear operation with optical phase-coherent spatial modes.

It should be noted, however, that due to the intrinsic loss of beam splitting and filtering, our proposal is not lossless for an arbitrary operator. Thus our approach would suffer from the efficiency for the cases that the insertion loss is critical, e.g. some quantum photonic applications. Theoretically, the lower bound of optimized transformation efficiency is $\sim 1 / \sqrt{N}$ and the achieved value is $\sim 0.8 / \sqrt{N}$ in experiments. In practice, the efficiency depends on how to map the matrix elements into the phase grating patterns and there is still some space for more improvement.

\section{Acknowledgments}

Acknowledgements This work was supported by the National Key Research and Development Program of China (2017YFA0303700), the National Natural Science Foundation of China (Grant No. 61875101 and 61621064), the Royal Society Research Professorships (RP150122), Beijing Innovation Center for Future Chip and Beijing academy of quantum information science. The authors would like to thank Dr. Yu Wang for valuable discussions and helpful comments and Prof. David Miller for a helpful correspondence.

\section{References}

[1] Knill E, Laflamme R, Milburn GJ 2001 A scheme for efficient quantum computation with linear optics nature. 409 46-52

[2] Tillmann M, Dakić B, Heilmann R, Nolte S, Szameit A, Walther P 2013 Experimental boson sampling Nature Photonics 7 540-4

[3] Crespi A et al 2013 Integrated multimode interferometers with arbitrary designs for photonic boson sampling Nature Photonics 7 545-9 
[4] Broome MA et al 2013 Photonic boson sampling in a tunable circuit Science 339 794-798

[5] Spring JB et al 2013 Boson sampling on a photonic chip Science 339 798-801

[6] Spagnolo N et al 2014 Experimental validation of photonic boson sampling Nature Photonics 8 615-20

[7] Carolan J et al 2014 On the experimental verification of quantum complexity in linear optics Nature Photonics 8 621-6

[8] Carolan J et al 2015 Universal linear optics Science 349 711-716

[9] Wang $\mathrm{H}$ et al 2017 High-efficiency multiphoton boson sampling Nature Photonics 11361

[10] Rohde PP, Schreiber A, Stefaňák M, Jex I, Silberhorn C 2011 Multi-walker discrete time quantum walks on arbitrary graphs, their properties and their photonic implementation New Journal of Physics 13013001

[11] Harris NC et al 2017 Quantum transport simulations in a programmable nanophotonic processor Nature Photonics 11 447-52

[12] Rohde PP, Fitzsimons JF, Gilchrist A 2012 Quantum Walks with Encrypted Data Physical Review Letters 109150501

[13] Motes KR, Olson JP, Rabeaux EJ, Dowling JP, Olson SJ, Rohde PP 2015 Linear Optical Quantum Metrology with Single Photons: Exploiting Spontaneously Generated Entanglement to Beat the Shot-Noise Limit Physical Review Letters 114170802

[14] Zhuang L, Roeloffzen CGH, Hoekman M, Boller KJ, Lowery AJ 2015 Programmable photonic signal processor chip for radiofrequency applications Optica 2854

[15] Stabile R, Albores-Mejia A, Rohit A, Williams KA 2016 Integrated optical switch matrices for packet data networks Microsystems \& Nanoengineering 215042

[16] Shen Y et al 2017 Deep learning with coherent nanophotonic circuits Nature Photonics 11441

[17] Reck M, Zeilinger A, Bernstein HJ, Bertani P 1994 Experimental realization of any discrete unitary operator Phys Rev Lett $\mathbf{7 3} 58-61$

[18] Clements WR, Humphreys PC, Metcalf BJ, Kolthammer WS, Walsmley IA 2016 Optimal design for universal multiport interferometers Optica 31460

[19] Miller DAB 2013 Self-configuring universal linear optical component Photonics Research 11

[20] Annoni A et al 2017 Unscrambling lightautomatically undoing strong mixing between modesLight: Science \&) Applications 6 e17110

[21] Wang J et al 2018 Multidimensional quantum entanglement with large-scale integrated optics Science $\mathbf{3 6 0}$ 285-91

[22] Schlederer F, Krenn M, Fickler R, Malik M, Zeilinger A 2016 Cyclic transformation of orbital angular momentum modes New Journal of Physics 18043019

[23] Lukens JM, Lougovski P 2017 Frequency-encoded photonic qubits for scalable quantum information processing Optica 48

[24] Morizur J-F et al 2010 Programmable unitary spatial mode manipulation JOSA A $\mathbf{2 7}$ 2524-2531

[25] Labroille G, Denolle B, Jian P, Genevaux P, Treps N, Morizur J-F 2014 Efficient and mode selective spatial mode multiplexer based on multi-plane light conversion Optics Express 2215599

[26] Berkhout GCG, Lavery MPJ, Courtial J, Beijersbergen MW, Padgett MJ 2010 Efficient Sorting of Orbital Angular Momentum States of Light Physical Review Letters 105153601

[27] Leach J, Padgett MJ, Barnett SM, Franke-Arnold S, Courtial J 2002 Measuring the orbital angular momentum of a single photon Physical review letters
88257901

[28] Leonardo Neves, G. Lima, J. G. Aguirre Gómez, C. H. Monken, C. Saavedra, S. Pádua 2005 Generation of Entangled States of Qudits using Twin Photons Physical Review Letters 94100501

[29] A. Z. Khoury, L. E. Oxman, B. Marques, A. Matoso, S. Pádua 2013 Fractional topological phase on spatially encoded photonic qudits Physical Review A 87042113

[30] S. P. Walborn, D. S. Lemelle, M. P. Almeida, P. H. Souto Ribeiro 2006 Quantum Key Distribution with Higher-Order Alphabets Using Spatially Encoded Qudits Physical Review Letters 96090501

[31] A.A. Matoso, R.A. Ribeiro, L. E. Oxman, A. Z. Khoury, S. Pádua 2019 Fractional topological phase measurement with a hyperentangled photon source Scientific Reports 9577

[32] Gabriela B. Lemos, Rafael M. Gomes, Stephen P. Walborn, Paulo H. Souto Ribeiro, Fabricio Toscano 2012 Experimental observation of quantum chaos in a beam of light Nature Communications 31211

[33] Wang Y, Potoček V, Barnett SM, Feng X 2017 Programmable holographic technique for implementing unitary and nonunitary transformations Physical Review A 95033827

[34] Radwell N, Offer RF, Selyem A, Franke-Arnold S 2017 Optimisation of arbitrary light beam generation with spatial light modulators Journal of Optics 19095605

[35] Zhao P et al 2017 Measuring the complex orbital angular momentum spectrum of light with a mode-matching method Optics Letters 421080

[36] Kues M et al 2017 On-chip generation of high-dimensional entangled quantum states and their coherent control Nature $\mathbf{5 4 6} 622-6$ 